UCRL-ID--105666

\title{
A Computer Model for Analyzing Low-Yield Threshold Test Ban Treaties
}

DE9 1006222

\author{
Richard M. Bradford and Padmini R. Sokkappa \\ Systems Research Group \\ Lawrence Livermore National Laboratory
}

\begin{abstract}
With the ratification of the Threshold Test Ban Treaty (TTBT), there has been increased interest in lowering the nuclear test ban threshold from its present level of 150 kilotons and much debate about what this lower threshold should be. The uncertainties associated with verifying the TTBT, along with the added difficulties associated with lower yields, have aroused concern that a Low-Yield Threshold Test Ban Treaty (LYTTBT) may not be sufficiently verifisble. This report documents a computer model for analyzing the verifiability of LYTTBT options. The model systematically accounts for the uncertainty inherent in the treaty verification process. It helps the user to gain insights into the interactions among monitoring capability, beliefs about Soviet testing strategies, and the likelihood of missing violations or making false accusations of violations. The model can also account for possible Soviet evasion strategies that could systematically reduce the U.S. estimates of Soviet test yields. There are eight component.; of the model, each facilitating a different type of analysis. The components are: (1) Cavity Decoupling, (2) Verification Effectiveness, (3) Probability of Missed Violation, (4) Probability of False Accusation, (5) Detectable Violation, (6) Distinguishable Yield, (7) Required Monitoring Capability, and (8) Expected Utility. The Cavity Decoupling component allows the user to analyze the effects of evasion by cavity decoupling. The next six components address the verifiability of alternate LYTTBTs from different perspectives. Finally, the Expected Utility component computes the expected overall utility of each threshold option. Decision makers can use the various components of the model to study issues related to the verifiability of various LYTTBT options. Because the model is explicit and quantitative, it provides decision makers with rational, defensible analyses, which can be used as input when making important treaty and verification decisions.
\end{abstract}

\section{MASTER}




\section{Table of Contents}

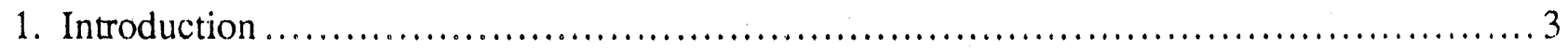

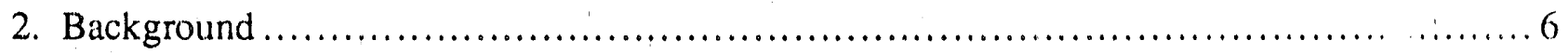

3. The Cavity Decoupling Component...................................................... 10

4. The Verification Components................................................... 12

5. The Expected Utility Component.............................................................19

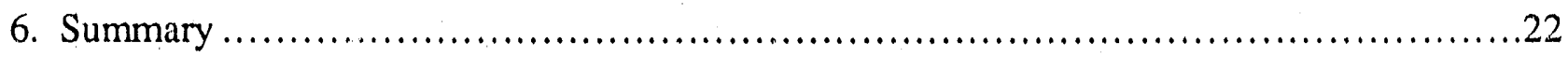

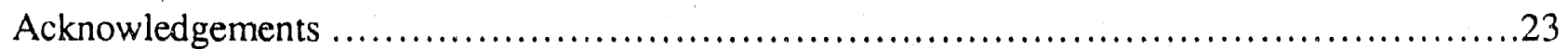

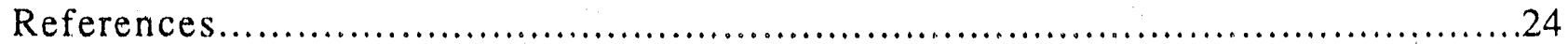

Appendix A: Instructions for Running the Software .....................................25

Appendix B: Formats of Input and Output Files......................................29 


\section{Introduction}

In September 1987, the U.S. and Soviet Union agreed to begin stage-by-stage negotiations of nuclear test limitation treaties, the ultimate objective being the complete cessation of nuclear testing. The first step in these negotiations was to be agreement on effective verification measures that would make possible the ratification of the US-USSR Threshold Test Ban Treaty of 1974 (TTBT) and the Peaceful Nuclear Explosions Treaty of 1976 (PNET). This was to be foilowed by negotiation of further intermediate limitations on nuclear testing, ultimately leading to a Comprehensive Test Ban Treaty. With the recent ratification of the TTBT and PNET, a critical issue facing DOE and the nation is what intermediate steps should be pursued. The options to be considered include lower yield thresholds and/or quotas on the frequency of tests at declared test sites. The uncertainties associated with verifying the TTBT, along with the added difficulties associated with lower yields, have aroused concern that a Low-Yield Threshold Test Ban Treaty (LYTTBT) may not be sufficiently verifiable.

This report documents a computer model for analyzing Low-Yield Threshold Test Ban Treaty (LYTTBT) options, with emphasis on verififiability. Earlier research on verifying treaty compliance, such as "Decision Framework for Evaluating Compliance with the Threshold Test Ban Treaty," 1 demonstrated the applicability of decision analysis models to verification issues. A similar decision analysis approach was used in the development of this model. The model systematically accounts for the various types of uncertainty inherent in the treaty verification process. It helps the user to gain insights into the interactions among monitoring capability, beliefs about Soviet testing strategies, and the likelihood of missing violations or making false accusations of violations. The model can also account for possible Soviet evasion strategies that could systematically reduce the U.S. estimates of Soviet test yields. The model has been implemented on a Macintosh computer, providing a flexible, easy to use, and comprehensive tool. It makes possible the rapid analysis of a variety of issues related to lower yield thresholds and enhances an analyst's capability to respond quickly to requests by the DOE Office of Arms Control (and others) for analysis of verification issues.

Verifiability of a threshold can be defined as the ability to distinguish between compliance and a "minimum significant violation" - the minilulum lev'el of testing abov the threshold by the Soviet Union that would $t$. detrimental to U.S. national security if the U.S. were constrained by the threshold. Verifiability is quantified by two measures, the probability of false accusation (challenging the Soviets when, in fact, the yield of the test was below the treaty limit) and the probability of missing a significant violation (failing to challenge when, in fact, a significant violation has occurred). Although it would be desirable to have low probabilities of false accusations and missed violations simultaneously, this is not always possible. Because of uncertainty in the monitoring and yield estimation process, there exists a tradeoff - the lower the probability of a false accusation, the higher the probability of missing a significant violation, and vice versa. The uncertainty is due to random effects in the monitoring process, as well as a lack of information about the relationship between monitoring data obtained for Soviet tests and the actual yields of these tests. Monitoring capability, which is characterized by the "F-factor," is defined in terms of this uncertainty. 
There are eight components of the model. Each facilitates a different type of analysis. The components are: (1) Cavity Decoupling, (2) Verification Effectiveness, (3) Probability of Missed Violation, (4) Probability of False Accusation, (5) Detectable Violation, (6) Distinguishable Yield, (7) Required Monitoring Capability, and (8) Expected Utility. The first of these allows analysis of the potential for evasion by cavity decoupling, the next six address verification capability, and the last allows an overall evaluation of threshold options. The components are designed to facilitate sensitivity analysis, thereby making it easy to evaluate the effects of alternative judgments about the input parameters. Results of each component are displayed graphically. The graphs and the data used to generate them can be saved in user-specified files.

The Cavity Decoupling component of the model computes the relationship between actual yields and apparent yields for a scenario involving evasion by cavity decoupling. This data can then be used as input to the other components of the model. Currently, cavity decoupling is the only evasion strategy explicitly addressed in the model. Other evasion strategies, such as material decoupling, can be taken into account when using the other model components, but the user must supply the necessary data.

The Verification Effectiveness component of the model computes the relationship between the probabilities of the two types of errors for a fixed threshold, significant violation level and monitoring capability. The Probability of False Accusation component and the Probability of Missed Violation component allow the user to specify the probability of one type of error and observe how the other error probability varies over the range of threshold options.

In the Detectable Violation component, the user specifies maximum acceptable probabilities of the two types of errors. The software then computes, for each threshold, the lowest violation yield that is detectable with the specified maximum acceptable probabilities of false accusation and missed violation. This component can be useful for gaining insights about what types of weapon developments can be inhibited by particular treaty thresholds and protocols. The Distinguishable Yield component is the colinterpart to the Detectable Violation component. The model computes, for each of several violation levels, the yicld that can be distinguished, with the specified maximum acceptable probabilities of the two types of errors, from the specified violation level. This is particularly useful for setting yield levels for the implementation of various monitoring systems.

Because the relationship between significant violation yield and treaty threshold varies with the different threshold options, a given monitoring capability might be satisfactory for some thresholds but not for others. The Required Monitoring Capability component allows the user to specify maximum acceptable probabilities of false accusations and missed violations. It then computes, for each threshold option, the F-factor, or monitoring capability, that would be required in order to meet the specified verification requirements.

As mentioned above, there is a tradeoff between the probabilities of the two types of errors. One may wish to combine these measures of verification effectiveness in such a way as to get relative overall measures of different treaty thresholds and protocols. These measures naturally depend on the relative utility values of false accusations and missed violations. They also depend on the utility associated with accepted compliance and detected violations for each threshold. The Expected Utility component allows the user to specify these utility values and compute, for each treaty threshold option, an expected overall utility. 
The next section provides some background material and related mathematical equations necessary to understand the following discussion of the individual components. The remainder of the report documents the individual components of the model, including their assumptions, logic, inputs and outputs. The appendices include descriptions of the input and output files and file formats, as well as brief instructions on how to run the software. 


\section{Background}

\subsection{Monitoring Capability}

Because of uncertainty and random effects in the monitoring and yield estimation process, the yield estimate obtained by the U.S. for a Soviet test may differ from the actual yield. First, there is uncertainty about the exact relationship between monitoring data obtained for Soviet tests and the actual yields of these tests. Second, because of random effects, two tests with the same actual yield may result in different monitoring data, giving rise to two different yield estimates. The relationship between actual yield and estimated yield is assumed to follow a lognormal probability distribution. This means that the logarithm of the estimated yield is a random variable whose probability density function is normal (or "bell-shaped"). The expected value of this random variable is the logarithm of the actual yield, and the variance is characterized by a quantity called the "F-factor." The F-factor provides a measure of the spread in the distribution of yield estimates. If the F-factor associated with a monitoring system and an actual yield is 2 , this means that, when a Soviet test device with an actual yield of $y$ is exploded, the yield estimate based on that monitoring system is equally likely to be above or below $y$, and that there is a 95 percent probability that the estimated yield will fall between $y / 2$ and $2 y$. A higher F-factor corresponds to a less effective monitoring system. The F-factor for a monitoring system may vary with actual yield, and is typically higher for lower yields. When using the computer model to do analysis, the user must provide, for each monitoring system under consideration, a file characterizing the F-factors for that monitoring system as a function of yield.

\subsection{Significant Violation}

As mentioned previously, verifiability is defined as the ability to distinguish between compliance and a minimum significant violation of the treaty. This minimum significant violation should represent the level of testing by the Soviet Union that would be detrimental to U.S. national security, given that the U.S. is abiding by the threshold. The determination of the minimum significant violation may be based on a number of factors, including military and political considerations. Violations are defined by both a test yield and a number of tests that must be performed at that yield. In some cases, a single test at a specified yield might not be considered significant, while several tests at that yield would be. Some components of the model require the user to specify treaty threshold options, and, for each option, an associated violation test yield, the number of tests that must be performed at that yield to constitute a significant violation, and the number of tests at or near the threshold that constitute the compliance scenario. These data are generally specified in a threshold/violation file, which is used as an input to the model components.

\subsection{Verification Process}

The model assumes that the monitoring of Soviet compliance with an LYTTBT would be a two step process. The two steps are: (1) estimate the yield of each Soviet test; and (2) based on the estimated yield, decide whether to act as if the Soviets are complying or to accuse the Soviets of violating the threshold. Because of the inherent uncertainty in yield estimation, it is possible that the U.S. could decide to accuse the Soviets of a violation, when in fact the actual test yield was 
below the treaty threshold. This is, of course, a false accusation. It is also possible that the Soviets could conduct a significant violation that does not result in yield estimates large enough to cause the U.S. to react. This would constitute a missed violation. The other two possible outcomes are challenged violation, when a violation draws an accusation, and accepted (unchallenged) compliance, when the Soviets comply and the U.S. does not make an accusation.

We assume that the decision of whether to make an accusation is based on whether the yield estimate falls above or below a predetermined level. We call this yield level that determines the U.S. response the "decision line." The probability of a false accusation can be made arbitrarily small by raising the decision line. However, raising the decision line increases the probability of missing a violation. Similarly, the probability of missing a violation can be made arbitrarily small by lowering the decision line, but this will increase the probability of a false accusation.

\subsection{Mathematical Relationships}

This section presents equations that define some of the mathematical relationships of interest in the model. The probabilities of false accusation and of missed violation will be defined in terms of the decision line, the treaty threshold, the F-factor, and the compliance and violation scenarios. It will also be shown how the decision line can be determined when the probability of false accusation or the probability of missed violation is specified. The following notation will be used in defining the equations:

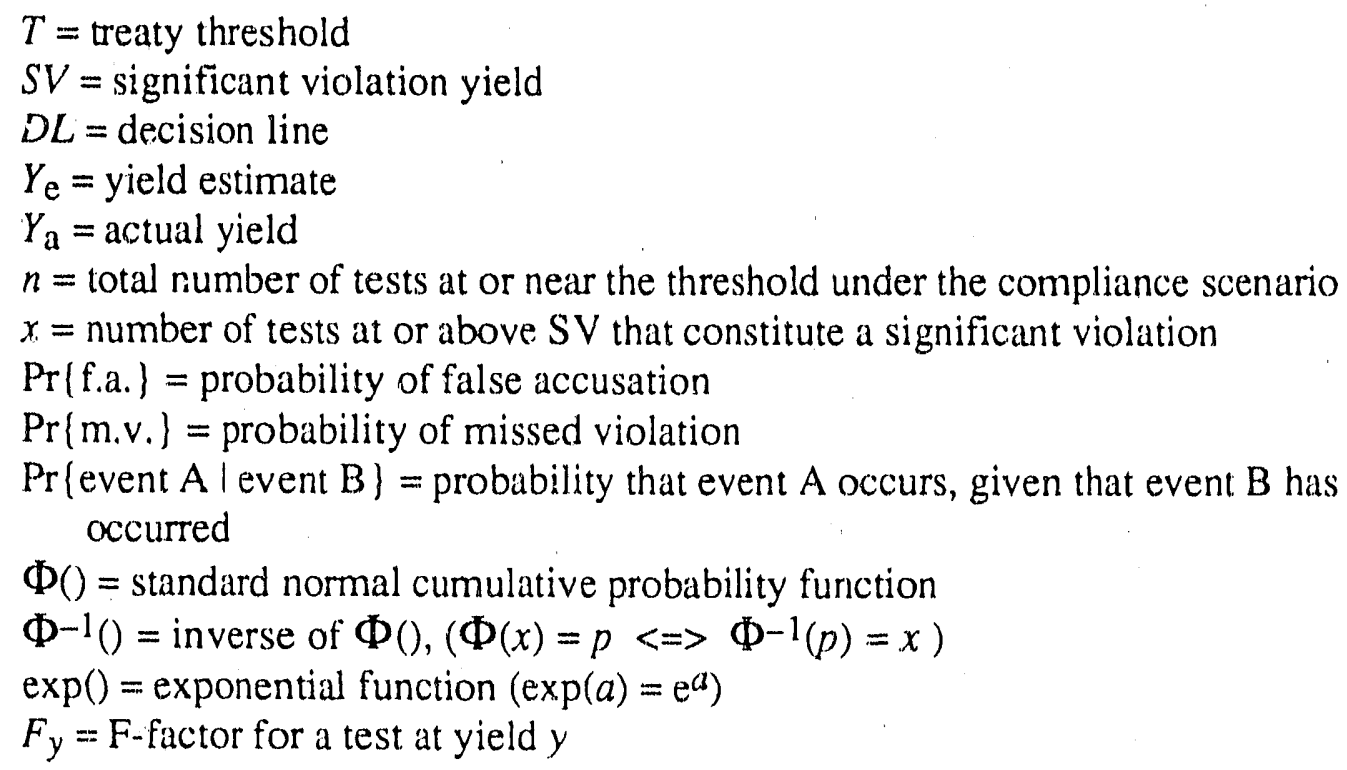

The probability of a false accusation is the probability that at least one yield estimate exceeds the decision line, given that all tests were at or below the threshold. Note that this probability is conditioned on the Soviets' complying with the treaty. Since yield estimates have a lognormal distribution,

$$
\operatorname{Pr}\{\text { f.a. }\}=\operatorname{Pr}\left\{Y_{\mathrm{e}}>D L \mid Y_{\mathrm{a}}=T\right\}=1-\Phi\left((\ln D L-\ln T) /\left(\ln F_{T} / 1.96\right)\right)
$$

for a single test. For a compliance scenario consisting of $n$ tests at or near the threshold, 


$$
\operatorname{Pr}(\text { f.a. })=1-\left[\operatorname{Pr}\left(Y_{\mathrm{e}}<D L \mid Y_{\mathrm{a}}=T\right\}\right]^{n}=1-\left[\Phi\left((\ln D L-\ln T) /\left(\ln F_{T} / 1.96\right)\right)\right]^{n} .
$$

Since there is a possibility of false accusation for each individual test in the compliance scenario, the probability of making at least one false accusation increases with $n$. Equation (1) can be used to compute the probability of a false accusation when the decision line is specified. When a desired probability of false accusation is specified, equation (1) can be rearranged to determine the appropriate decision line. This rearranging gives

$$
D L=\exp \left(\ln T+\Phi^{-1}\left([1-\operatorname{Pr}(\text { f.a. })]^{1 / n}\right) *\left(\ln F_{T} / 1.96\right)\right) .
$$

Finally, if both the probability of false accusation and the decision line are specified, and data on the relationship between yield and F-factor are available, an iterative search can be used to find the maximum threshold for which the specified probability of false accusation is not exceeded. Thus, there is a relationship among the threshold, the decision line, and the false accusation probability, such that if any two of these parameteri are specified, the third may be computed.

The probability of missing a violation is the probability that no yield estimate exceeds the decision line, given that $x$ tests were conducted at or above the significant violation yield. Note that this probability is conditioned on the Soviets' conducting a significant violation. The model assumes that this probability is based on the violation tests only, and does not consider the possibility of "catching" a violation scenario by misclassifying a legitimate test as a violation. For more discussion on this topic, see "Alternative Interpretations of TTBT Monitoring Effectiveness"2. For a violation consisting of a single test,

$$
\operatorname{Pr}\left(\mathrm{m} . \mathrm{v}_{\mathrm{v}}\right)=\operatorname{Pr}\left\{Y_{\mathrm{e}}<D L \mid Y_{\mathrm{a}}=S V\right)=\Phi\left((\ln D L-\ln S V) /\left(\ln F_{S V} / 1.96\right)\right) .
$$

For a violation consisting of multiple tests,

$$
\operatorname{Pr}\left(\mathrm{m} . \mathrm{v}_{\mathrm{v}}\right)=\left[\operatorname{Pr}\left\{Y_{\mathrm{c}}<D L \mid Y_{\mathrm{a}}=S V\right\}\right]^{x}=\left[\Phi\left((\ln D L-\ln S V) /\left(\ln F_{S V} / 1.96\right)\right)\right]^{x}
$$

This equation can be used to compute the probability of missing a violation when the decision line is specified. By rearranging equation (3), we can determine the decision line that leads to a specified probability of missed violation. This rearranging gives

$$
D L=\exp \left(\ln S V+\Phi^{-1}\left([\operatorname{Pr}(\text { m. v. })]^{1 / x}\right) *\left(\ln F_{S V} / 1.96\right)\right) .
$$

For a specified decision line and probability of false accusation, and given data on the relationship between yield and F-factor, an iterative search can be conducted to find the minimum violation yield for which the specified probability of missing a violation is not exceeded. Thus, as with the probability of false accusations, there is a relationship among the decision line, the violation yield, and the probability of missing a violation, such that if any two of these parameters are specified, the third may be computed.

As a result of these equations, it is clear that, for a given threshold and significant violation, there is a direct relationship between the probability of a false accusation and the probability of at 
missed violation. A specified probability of false accusation determines the desired decision line, which in turn determines the probability of missing a violation, and vice versa.

The equations given in this section do not address the potential effects of evasion scenarios. If the Soviet Union tries to evade a treaty, the differences between the actual ylelds of Soviet violation tests and the apparent yields of these tests given the evasion scenario must be taken into account. This is done by replacing the actual violation yield in the equations with the associated apparent yield under the evasion scenario. The Cavity Decoupling component of the model, described in the next section, computes these apparent yields for evasion scenarios involving cavity decoupling. 


\section{The Cavity Decoupling Component}

The Cavity Decoupling component of the Low Yield computer model facilitates analysis of the ability of the Soviet Union to "decouple" a nuclear explosion by placing the device in an underground cavity, Decoupling reduces the amount of explosive energy transmitted in the form of seismic waves. Thus, decoupling allows a country to appear to abide by the treaty while actually conducting tests with yields above the treaty threshold.

The degree of decoupling that can be obtained depends on the yield of the explosion and the size of the underground cavity. This level of decoupling is represented by a decoupling factor. The decoupling factor is equal to the ratio of the apparent yield to the actual yield. It is a decreasing furction of the scaled radius of the cavity, where

$$
\text { scaled radius }=r / Y_{\mathrm{a}}{ }^{1 / 3}
$$

with $Y_{\mathrm{a}}$ denoting the actual yield of the test device, and $r$ being the actual radius of the cavity, measured in meters. Thus if a test device with an actual yield of $Y_{\mathrm{a}}$ is exploded in a spherical cavity with radius $r$ meters, the apparent yield of this explosion is:

$$
y_{\text {apparent }}=Y_{\mathrm{a}} * \mathrm{DF}\left(r / Y_{\mathrm{a}}^{1 / 3}\right),
$$

where DF is the decoupling factor function. The model requires the user to provide a file containing data that characterize the decoupling factor as a function of scaled radius. The data will depend on assumptions about the material in which the cavity is constructed.

There is some uncertainty about the maximurn size cavity that the Soviet Union is able or willing to construct without detection. The model requires the user to specify the maximum cavity radius (in meters) that the Soviets might be expected to use. The Cavity Decoupling component then uses this maximum cavity size, along with the decoupling factor data, to compute the apparent yield for each of a range of actual yields. Results are presented as a graph showing apparent yield as a function of actual yield. Up to five cases can be displayed on one graph, and this graph can be saved in a user-specified file.

The data generated for each case run by the Cavity Decoupling component is saved in an individual file, which, if desired, can then be used as input to the other components of the model. It is assumed that the yield estimate for a decoupled explosion has the same uncertainty as the yield estimate for a test whose actual yield is the decoupled apparent yield. Thus, the other components of the model account for cavity decoupling by replacing the actual yield of tests in violation of the threshold with the associated apparent yield under the cavity decoupling scenario (i.e. substituting $y_{\text {apparent }}$ for $Y_{\mathrm{a}}$ in equations (1) through (4)).

Although cavity decoupling is the only evasion scenario explicitly addressed in the model, other methods of evasion, such as material decoupling, may be considered when running the other components of the model. In these cases, the Cavity Decoupling component is not used. Instead, 
the user ruust supply a file containing data that characterize the relationship between actual yield and apparent yield under the evasion scenario. The format of this file is defined in Appendix B under the heading "Evasion Potential File." 


\section{The Verification Components}

The model contains six components that may be used to evaluate the verifiability of alternate LYTTBTs from different perspectives - (1) Verification Effectiveness, (2) Probability of Missed Violation, (3) Probability of False Accusation, (4) Detectable Violation, (5) Distinguishable Yield, and (6) Kequired Monitoring Capability. In each of these components, a different subset of the variables - probability of false accusation, probability of missed violation, threshold, violation yield, and F-factor - in Equations (1)-(4) of Section 2 are specified as input parameters. The appropriate equations are then used to compute the value of the remaining variable. The values used for the input parameters may represent the user's best judgment about the actual (or desired) state of affairs, or they may simply be values chosen for the purpose of doing exploratory calculations. The computed value has one of two interpretations: it may be a consequence of the values of the input parameters, as is the case for the Verification Effectiveness, Probability of False Accusation, and Probability of Missed Violation components; or, it may be the value required in order for the ipecified values of the input parameters to be consistent, as is the case for the Detectable Violation, Distinguishable Yield, and Required Monitoring Capability components.

Table 1 indicates the computed value and the source of the input parameters for each of the six components. The labels FA, MV, T, V, and F refer, respectively, to probability of false accusation, probability of missed violation, threshold violation yield, and F-factor. A U entry in the table indicates the parameter is a user-defined c.unstant. An $\mathrm{R}$ indicates that the program uses a predetermined range of values for the parameter, and an $\mathrm{L}$ indicates that the parameter values are specified in a user-supplied file. A C indicates the value that is computed. Each component produces a graph showing the computed value as a function of one of the input parameters. An X indicates this parameter, which is plotted on the horizontal axis of the graph. The computed value is plotted on the vertical axis.

Table 1. The computed value and source of the input parameters for each of the six verification components.

Parameter

\begin{tabular}{|c|c|c|c|c|c|}
\hline Component & FA & MV & $\mathrm{T}$ & V & $\mathrm{F}$ \\
\hline Verification Effectiveness & $R, X$ & $\mathrm{C}$ & U & U & $\mathrm{L}$ \\
\hline Prob. of False Accusation & $\mathrm{C}$ & $\mathrm{U}$ & L, $X$ & $\mathrm{~L}$ & $\mathrm{~L}$ \\
\hline Prob. of Missed Violation & U & $\mathrm{C}$ & L, X & $\mathrm{L}$ & $\mathrm{L}$ \\
\hline Dectectable Violation & U & $\mathrm{U}$ & $R, X$ & $\mathrm{C}$ & $\mathrm{L}$ \\
\hline Distinguishable Yield & $\mathrm{U}$ & U & $\mathrm{C}$ & $R, X$ & $\mathrm{~L}$ \\
\hline F-Factor & U & U & L, $X$ & $\mathrm{~L}$ & $\mathrm{C}$ \\
\hline
\end{tabular}


Zach component allows up to five cases to be analyzed, overlaying the results of each case on the same graph. These graphs and the data used to generate them may be saved in userspecifiec files. The formats or these files are described in Appendix B. In the remainder of this section, we discuss each of the six components that address verification capability.

The Verification Effectiveness component of the model computes the relationship between the probability of a missed violation and the probability of a false accusation. The user must speciry the theshold of the LYTTBT, the number of tests at or near the threshold under the compliance scenario, and the significant violation (both yield level and the number of tests at that yield) ass'sciated with the given threshold. The user must also supply an F-factor file containing Ffactors as a function of yield for the monitoring system under consideration. The format of this file is described in .Appendix B. The prugram uses false accusation probabilities ranging from 0.01 to 0.99 and computes the associated missed violation probabilities. For each false accusation probabilicy, the program first computes a decision line, using equation (2), and then uses this decision line and equation (3) to compute the associated probability of missing a violation. A graph showing $\operatorname{Pr}$ (missed violation\} as a function of $\operatorname{Pr}$ \{false accusation\} is used to present the results. An example is shown in Figure 1.

The Protability of Missed Vinlation component computes the probability of missing a significant violation, given a specified prohability of false accusation, for each threshold option. The user specifies the desired probability ' ' $\mathrm{f}$ false accusation and supplies an F-factor file. The user must also supply threshold and violain data in a threshold/violation file, which lists threshoid options, and, for each threshold option, the number of tests comprising the compliance scenario and the associated minimum significant vioiation (yield and number of tests). The format of this file is described in Appendix B. The mocel takes each threshold in the threshold/violation file and computes the decision line, using equation (2), that results in the specified probability of ialse accusation. The model then uses this decision line and equation (3) to calculate the probability of missing a significant violation. Results are displayed as a graph showing the probability of missed violation as a function of threshold. An example is shown in Figure 2.

The Probability of False Accusation component computes the probability of false accusation, given a specified probability of missed violation, for each threshold option. The user specifies the desired probability of missing a significant violation, and supplies a threshold/violation file and an F-factor file. The model takes each threshold in the threshold/violation file and computes the decision line, using equation (4) that results in the specified probability of missed violation. The model then uses this decision line and equation (3) to calsulate the probability of a false accusation. Results are displayed as a graph showing the probability of fa'se accusation as a function of threshold.

The Detectable Violation component of the model computes, for each of a range of thresholds, the minimum violation yield that can be detected with specified maximum acceptable probabilities of missed violations and of false accusations. Since a significant violation is defined by $w$ th a yield and the number of tests conducted, the model requires the user to specify the number of violation tesis. The user must also specify the number of compliance tests and supply an F-factor file. For thresholds ranging from 5 to 150 kilotons in 5 - $\mathrm{kt}$ increments, the model uses equarion (2) to compute the decision line that results in the specified probability of false accusation. 


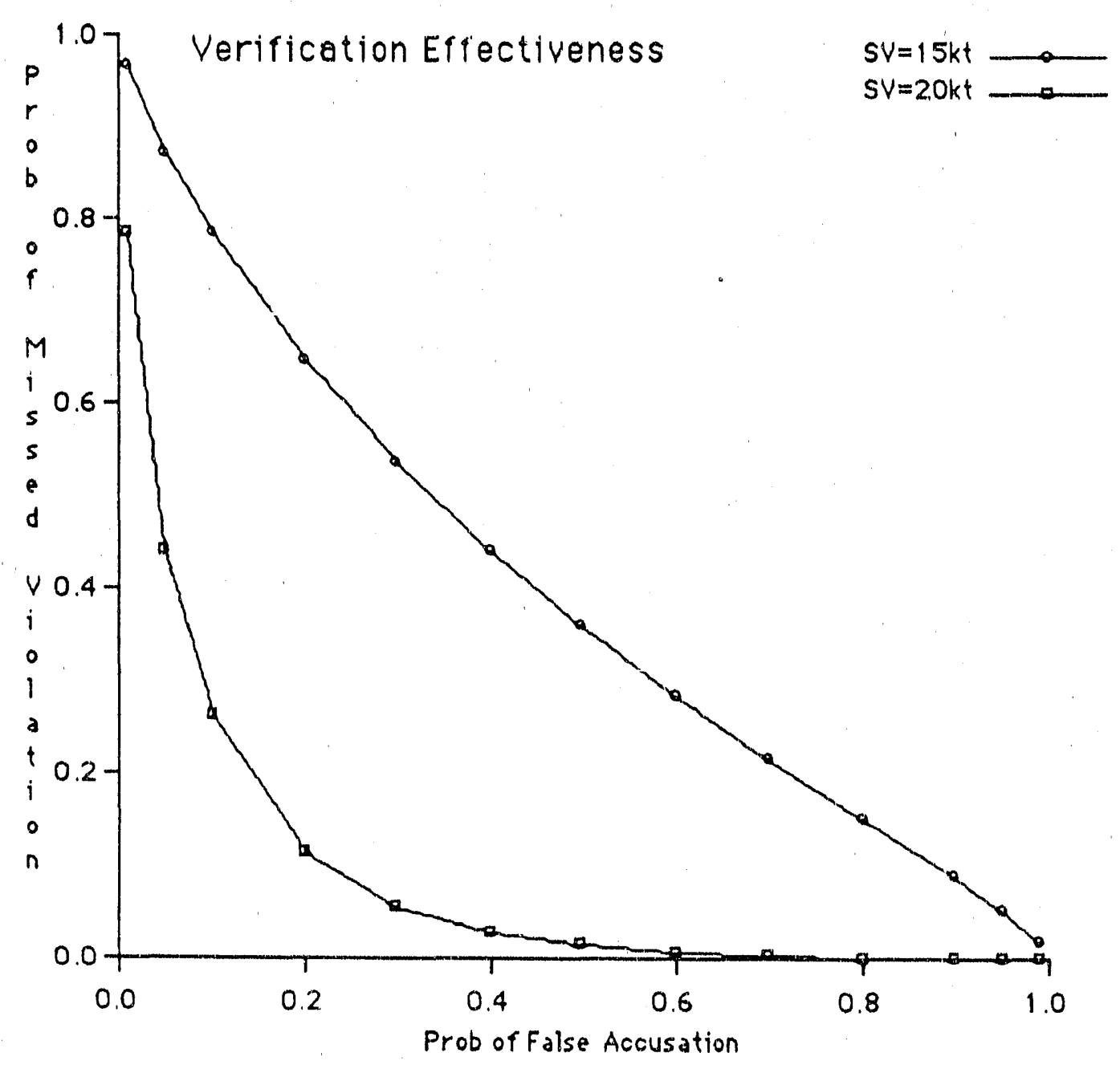

Figure 1. The Verification Effectiveness component computes the relationship between probability of false accusation and probability of missed violation for a given threshold, associated significant violation, and file of F-factors. This example uses illustrative data. The threshold used is $10 \mathrm{kt}$, and two case: are shown, one with the significant violation yield defined as $15 \mathrm{kt}$ and one with it defined as $20 \mathrm{kt}$. Up to five cases can be plotted on the same graph. The graph and the data used to generate it can be saved in user-specified files. 
10-m Cavity $\longrightarrow-\infty$
No Eyasion

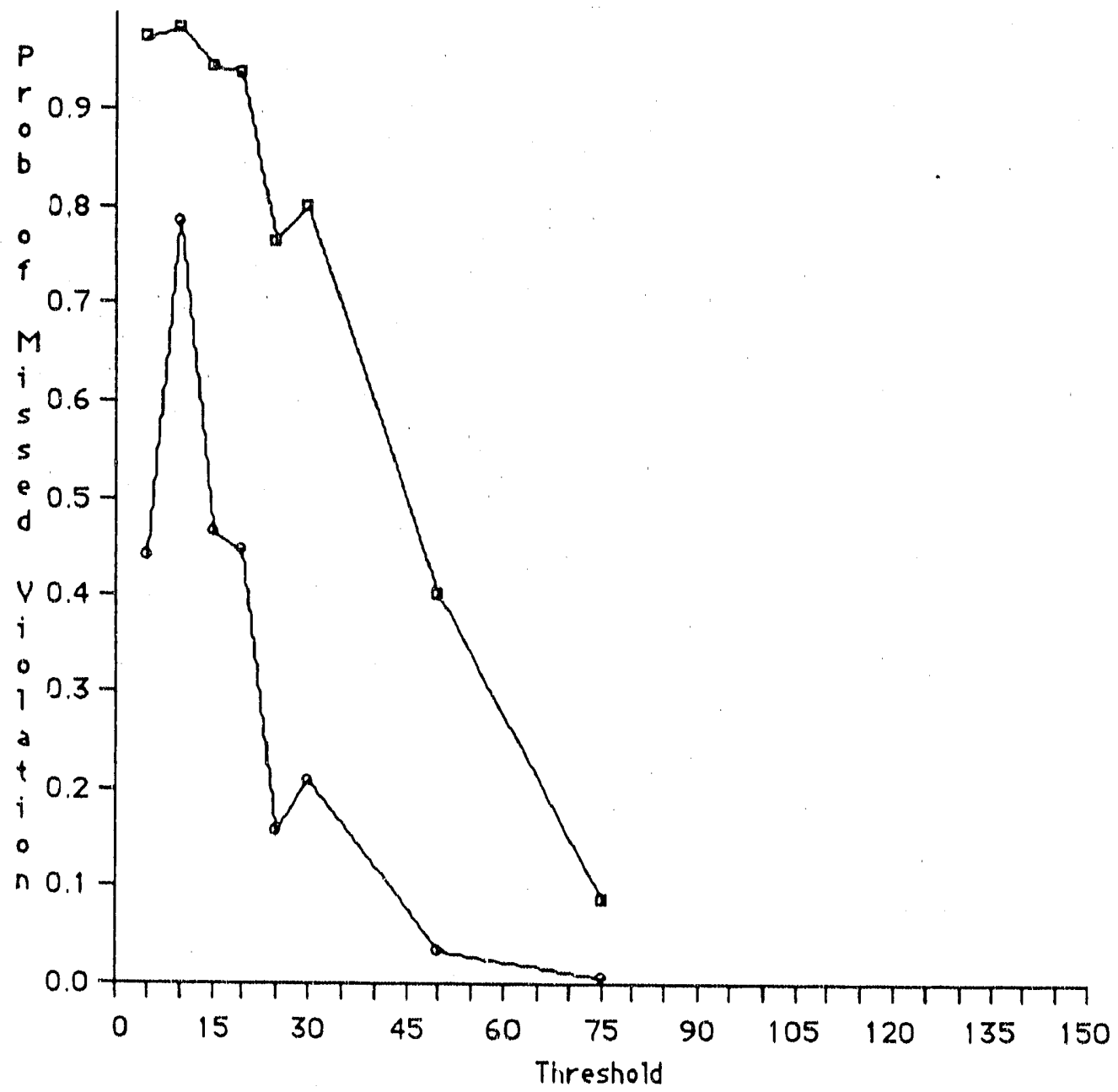

Figure 2. The Probability of Missed Violation component computes the relationship between threshold and probability of missed violation, given a probability of false accusation, F-factors, and pairs of thresholds and significant violations. The shapes of these curves are highly dependent on the threshold/violation pairs used. This example uses illustrative data. Two cases are shown, one without evasion and one with cavity decoupling. Up to five cases can be plotted on the same graph. The graph and the data 'ised to generate it can be saved in user-specified files. 
Using this decision line and the relationship between yield and F-factor, the program then uses equation (3) and does an iterative search for the minimum violation yield for which the probability of missed violation does not exceed the specified level. Results are preserited as a graph showing the detectable violation yield as a function of threshold.

The Distinguishable Yield component of the model computes, for each of a range of violation levels, the yield level that can be distinguished from the violation level with specified maximum acceptable probabilities of missed violations and of false accusations. As is the case with the Detectable Violation component, the user must specify the number of compliance and violation tests, and must also provide an F-factor file. For violation yields ranging from 10 to 300 kilotons in 10-kt increments, the model uses equation (4) to compute the decision line that results in the specified piobability of missed violation. Using this decision line and the relationship between yield and F-factor, the program then does an iterative search, using equation (1), for the maximum yield level for which the specified probability of false accusation is not exceeded. Results are presented as a grapi showing the distinguishable yield as a function of violation yield. An example is shown in Figure 3.

The Required Monitoring Capability component of the model computes, for each threshold option, the F-factor, or monitoring capability, that would be required to meet a specified verification requirement. The user defines this verification requirement by specifying maximum acceptable probabilities of missed violations and of false accusation. The user must also supply a threshold/violation file. An import it simplifying assumption, used only in this component of the model, is that the $\mathrm{F}$-factor is the same $\mathrm{f}, \mathrm{r}$ the threshold as for the violation yield. Combining equations (2) and (4) gives

$$
\begin{aligned}
& \left.\exp \left[\Phi^{-1}([1-\operatorname{Pr} \text { (f.a. })]^{1 / n}\right) *\left(\ln F_{T} / 1.96\right)+\ln T\right]=D L \\
& =\exp \left[\Phi^{-1}\left([\operatorname{Pr}(\text { m.v. }]]^{1 / x}\right) *\left(\ln F_{S V} / 1.96\right)+\ln S V\right] .
\end{aligned}
$$

Letting $F=F_{T}=F_{S V}$ and simplifying this equation results in

$$
\left[\Phi^{-1}\left([1-\operatorname{Pr}\{\text { f.a. }\}]^{1 / n}\right)-\Phi^{-1}\left([\operatorname{Pr}(\text { m.v. }\}]^{1 / x}\right)\right] * F=1.96 *(\ln S V-\ln T) \text {. }
$$

Using the specified probabilities of false accusation and missed violation, this equation is used to compute the required F-factor for each threshold option and its associated significant violation. Results are displayed as a graph showing the required F-factor as a function of threshold. An example is shown in Figure 4.

The six components discussed above can be used to analyze verification capability from a number of perspectives, but do not provide a measure of the overall desirability of alternate threshold options. The Expected Utility component, described in the next section, uses the same concepts as the verification components, plus additional judgments by the user or policy maker, to determine the expected overall utility for each treaty threshold. This allows the user to compare different threshold options on the basis of their relative utilities. 


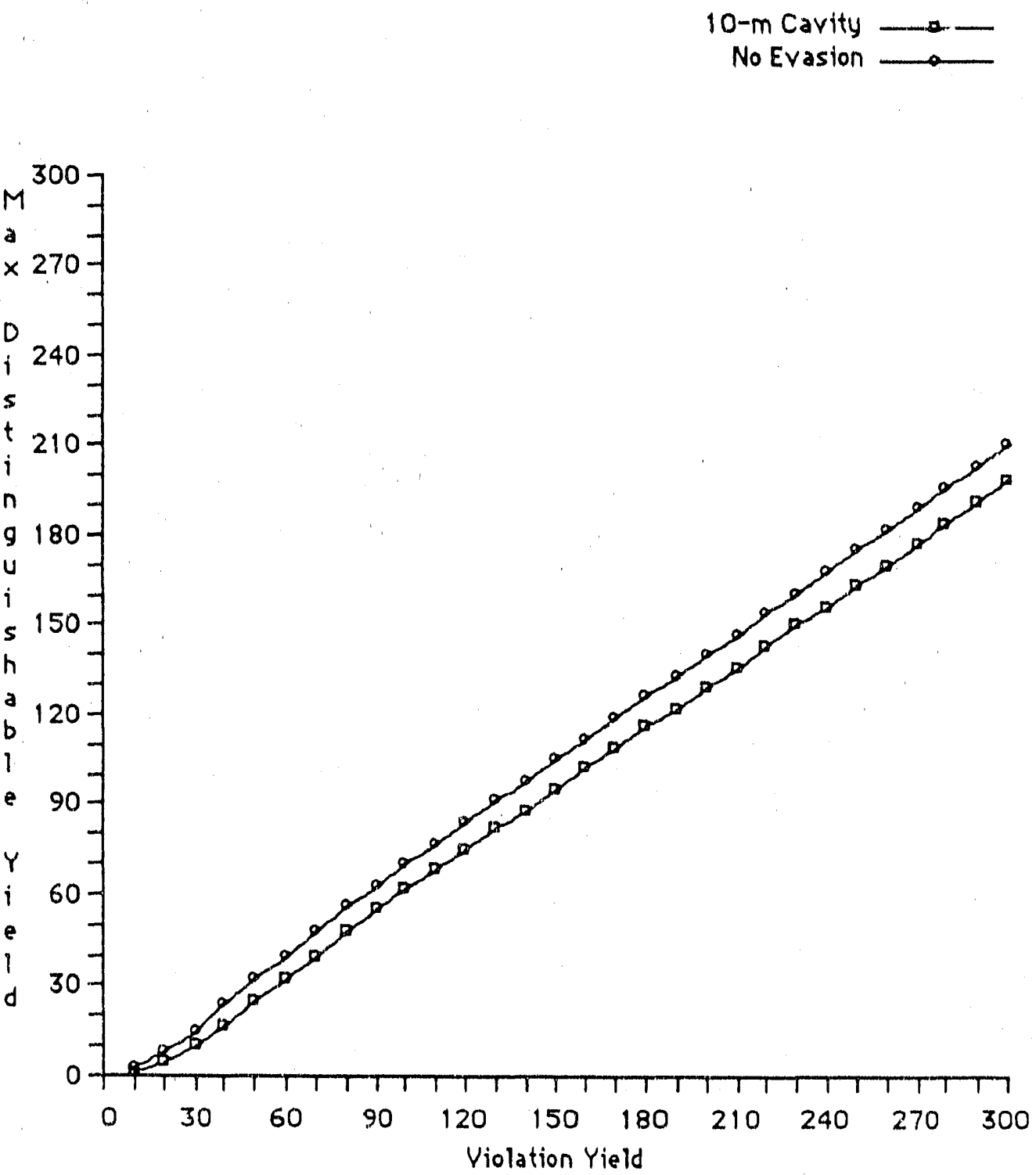

Figure 3. The Distinguishable Yield component computes the relationship between violation yieid and the largest threshold yield distinguishable from that violation yield, subject to specified probabilities of false accusation and missea violations. This example uses illustrative data. Up to five cases can be plotted on the same graph. Two cases are shown above. One assumes cavity decoupling using a 10-meter cavity; the other assumes no evasion. The graph and the data used to generate it can be saved in user-specified files. 


\section{Required Monitoring Capobility}

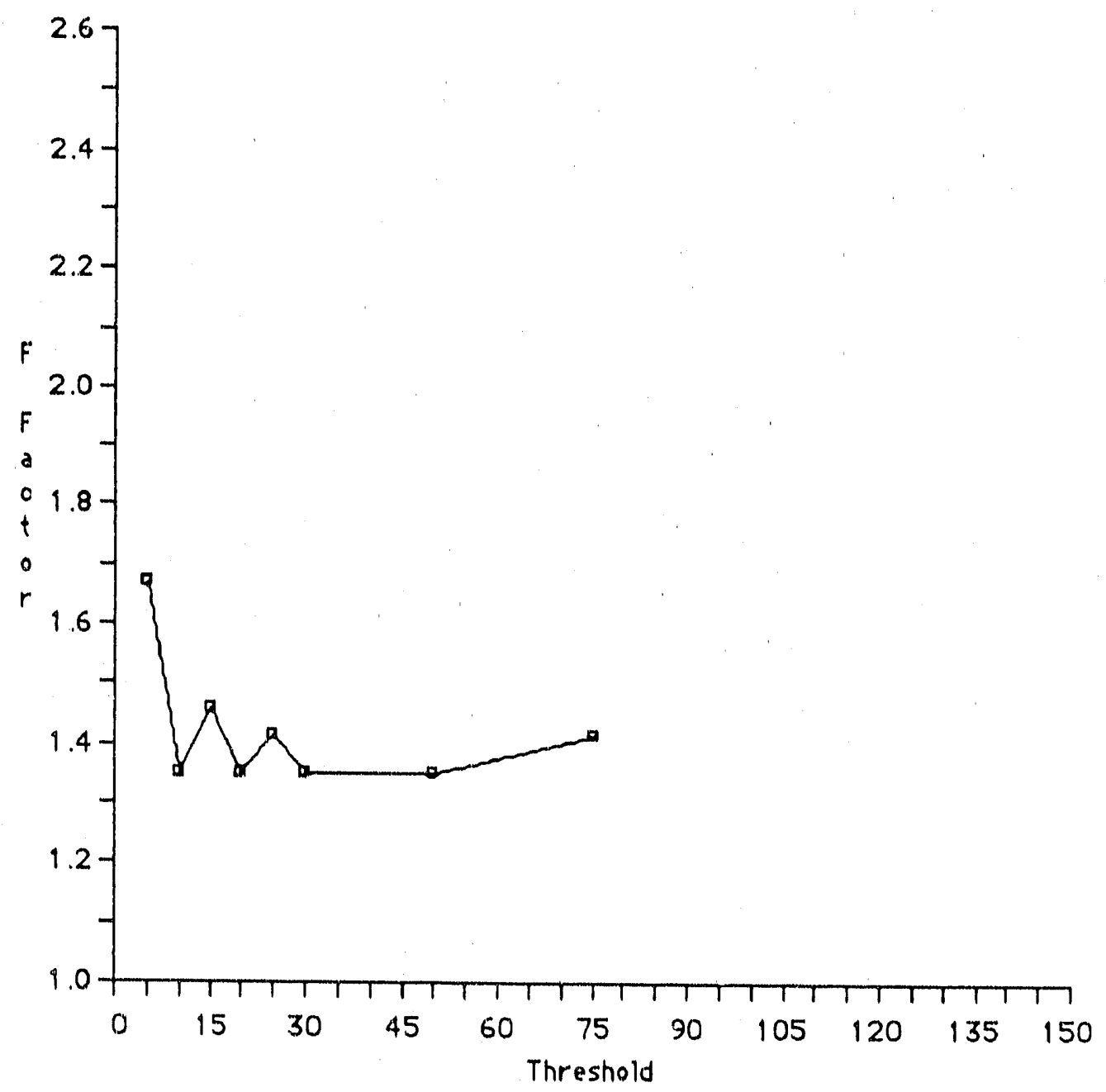

Figure 4. The Required Monitoring Capability component computes, for each threshold option, the F-factor, or monitoring capability, that would be required in order to meet specified maximum acceptable probabilities of missed violations and of false accusation. The shape of this curve is highly dependent on the threshold/significant violation data used. This example uses the illustrative data. Up to five cases can be plotted on the same graph. The graph and the data used to generate it can be saved ir user-specified files. 


\section{The Expected Utility Component}

The Expected Utility component of the model computes, for each threshold option, the expected overall utility of that threshold, based on user-provided relative utility values for each of the possible outcomes - accepted compliance, missed violation, false accusation and challenged violation - at each threshold, the policy maker's prior probabilities of compliance and violation, the computed conditional probabilities of missing a violation and making a false accusation.

The model requires the user to supply a utility data file containing a set of threshold options and, for each threshold, an associated significant violation yield, the number of tests in the compliance and significant violation scenarios, a prior probability of compliance, and utility values for each of the four possible outcomes. The format of this file is specified in Appendix B. As in previous components, the user must also supply an F-factor file for the monitoring system that will be used.

As discussed in Section 4, there is a tradeoff between the probability of a false accusation and the probability of a missed violation. Each decision line corresponds to a different pair of values for these error probabilities. Based on the relative utilities of the possible outcomes, as well as on the policy maker's prior probabilities of compliance and violation, the model calculates, for each threshold, the decision line that maximizes the expected overall utility.

In order to give a detailed description of the calculations performed by this component of the model, some additional notation is necessary. For a particular threshold, let

$\pi_{\mathrm{c}}=$ Policy maker's prior probability of Soviet compliance

$\pi_{\mathrm{v}}=1-\pi_{\mathrm{c}}=$ Policy maker's prior probability of Soviet vioiation

$u_{\mathrm{fa}}=$ Utility value associated with false accusation

$u_{\mathrm{ac}}=$ Utility value associated with accepted compliance

$u_{m v}=$ Utility value associated with missed violations, and

$u_{\mathrm{cv}}=$ Utility value associated with challenged violations.

Using this notation and the notation defined in Section 2, and recalling that the probability of false accusation and the probability of missed violation, as defined in Section 2, are conditioned on compliance and violation, respectively, the expected utility of a particular threshold is

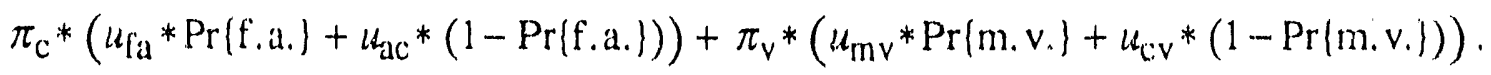

For a specified decision line, equations (1) and (3) can be used to compute $\operatorname{Pr}\left\{f_{\text {a. }}\right\}$ and $\operatorname{Pr}(m$. v. , respectively. For each threshold, the model use's the data on the associated violations and the F-factors, and does an iterative search for the decision line that maximizes the expected overall utility. The component then displays a graph of maximum expected utility versus threshold.

Up to five cases can be analyzed and displayed on the same graph. An examrle graph is shown in Figure 5. The graph and the data used to generate it can be saved in user-specified files. 
In addition, for each case analyzed, an individual file is created. These files contain the threshold options and, for each option, the expected overall utility, the decision line that results in this expected utility, and the resulting probabilities of false accusation and missed violations. 


\section{Overall Expected Utility}

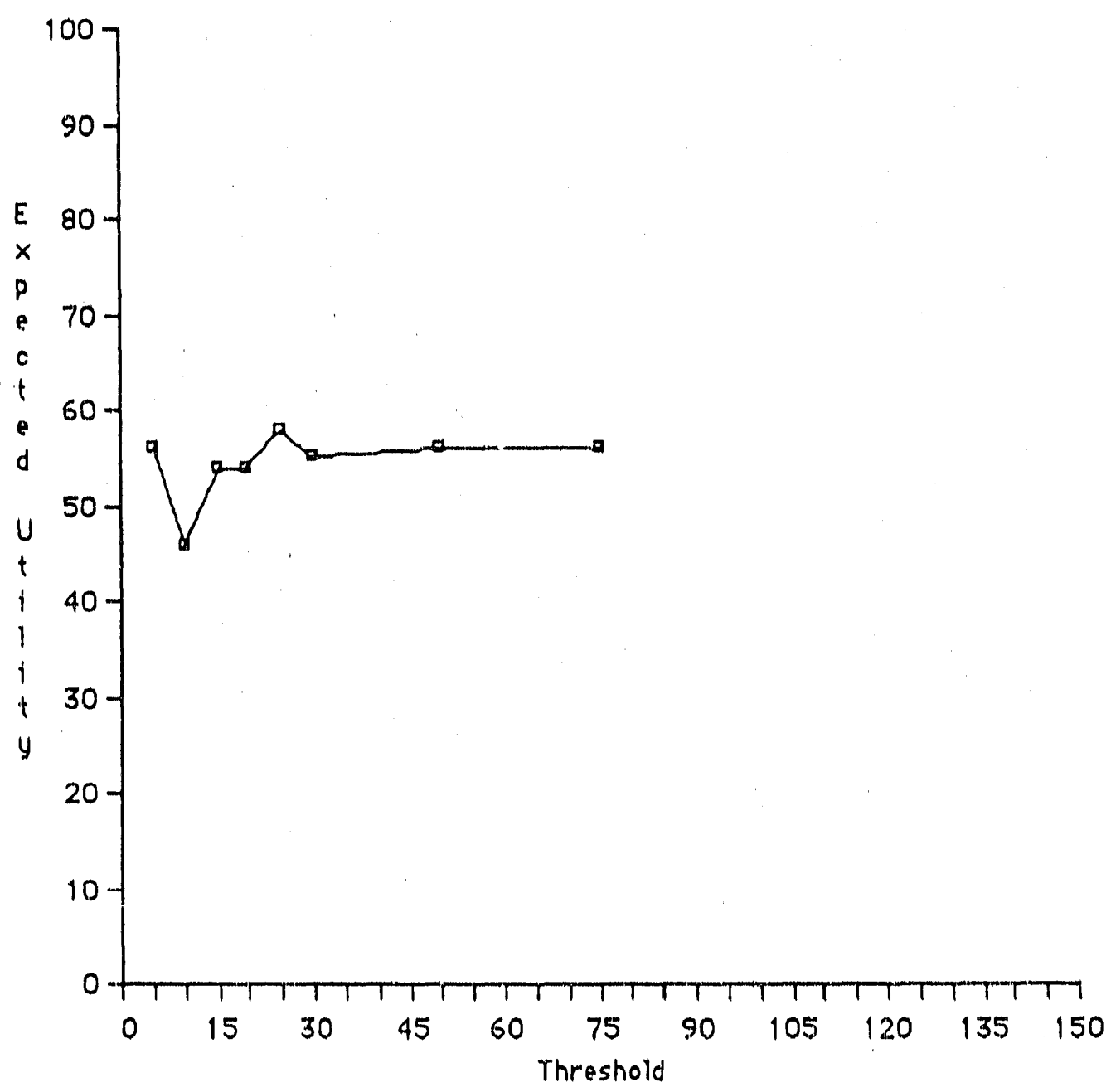

Figure 5. The Expected Utility component computes relative utilities of various threshold options. These relative utilities depend on judgments about what constitutes a significant violation of a given threshold, about the likelihood of such a violation, and about the utility values associated with the outcomes of accepted compliance, false accusation, missed violation, and challenged violation for each of a range of thresholds. The results also depend on the F-factors associated with various yields. Illustrative data are used in this example. Up to five cases can be plotted on the same graph. The graph and the data used to generate it can be saved in user-specified files. 


\section{Summary}

The model contains eight components, each providing a different analysis capability. The Cavity Decoupling component allows the user to analyze the effects of evasion by cavity decoupling. The six verification components address the veriflability of alternate LYTYBTs from different perspectives. Finally, the Expected Utility component computes the expected overall utility of each threshold option.

Decision makers can use the various components of the model to study issues related to the verifiability of various LYTTBT options. This can be done in several ways. For example, one cun specify the conditions of a treaty and use the model to assess its verifiability, or one can specify verification standards and use the model to determine conditions under which these standards can or cannot be met. Because the model is explicit and quantitative, it provides decision makers with rational, defensible analyses, which can be used as input when making important treaty and verification decisions. 


\section{Acknowledgements}

The authors would like to thank R. Scott Strait and John J. Zucca for their guidanco and assistance, and for editing various drafts of this report. We also wish to acknowledge the continued support and direction of the Department of Energy's Office of Arms Control and Willard J. Hannon of the Lawrence Livermore Treaty Verification Program. The computer model was implemented on the Macintosh computer by Betsy Hickman. 


\section{References}

1. Judd, B. R., R. S. Strait, L, W. Younker, P. C. Meagher, W. J. Hannon, Jr., and A. Sicherman (1988), Decision Framework for Evaluating Compliance with the Threshold Test Ban Treaty, Lawrence Livermore National Laboratory, Livermore, CA, UCRL53830 .

2. Bradford, R. M., and R. S. Strait (1990), Alterr ative Interpretations of TTBT Monitoring Effectiveness, Lawrence Livermore National Laboratory, Livermore, CA, UCRL-ID104105 . 


\section{Appendix A: Instructions for Running the Software}

The software is designed to run on an Apple Macintosh using MultiFinder. The software will run without MultiFinder, but anorralies, such as the disabling of double-clicking are likely to occur. These instructions assume that the user has already prepared input files as described in Appendix B. If this is not the case, the lllustrative data furnished with the model may be used.

"The icon for the model is labelled "Low Yield Model." The application is started by doubling clicking on the icon, or by selecting it and choosing "Open" from the "File" menu. When the application is running, the Menu Headings will be "File," "Edit," "Analysis," and "Options." The items in the "Edit" menu are disabled. The same is true of the items in the "Fille" menu, except for "Quit," which is self-explanatory. Below are diagrams of the "Analysis" menu and the "Options" menu.
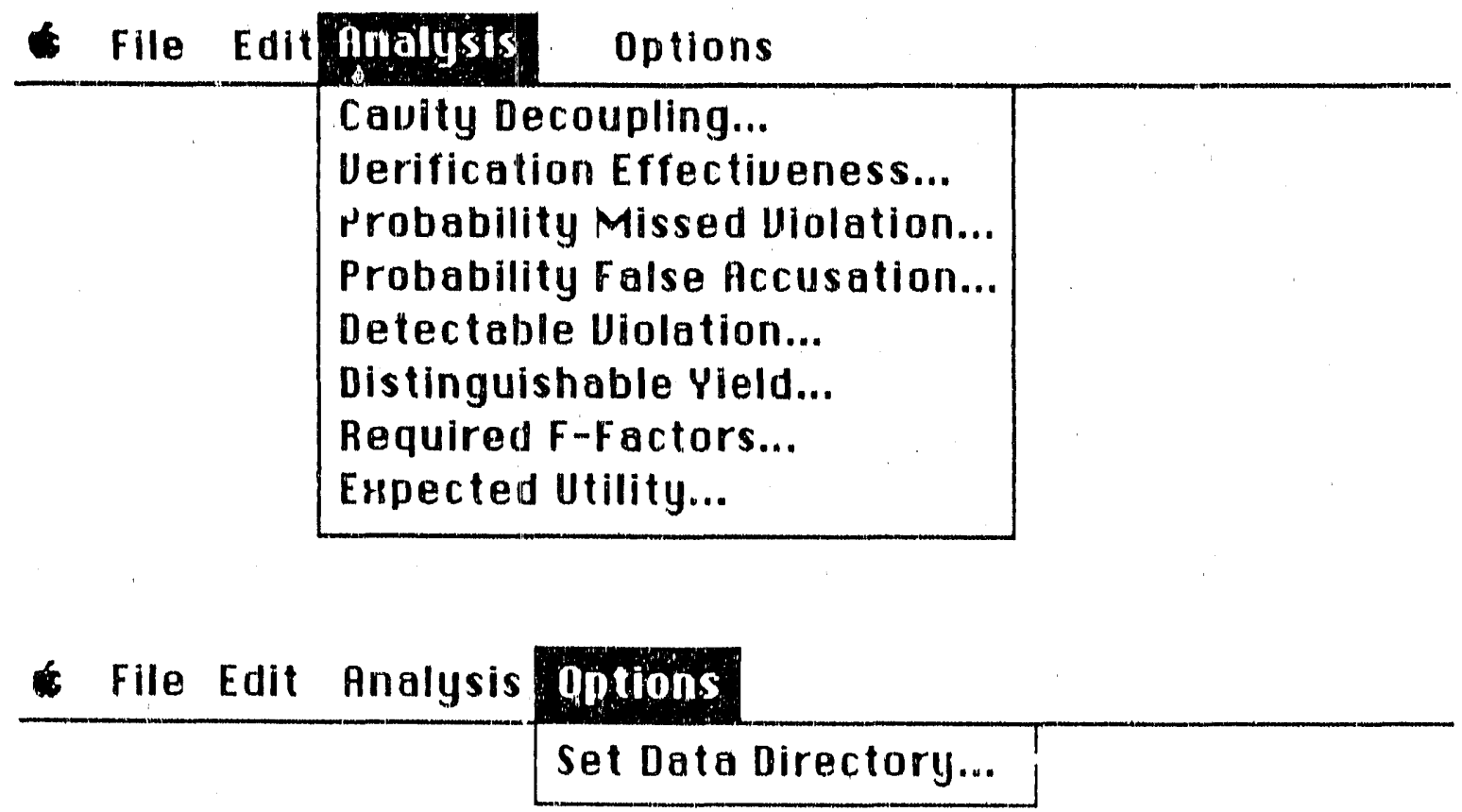

The "Set Data Directory" function under the "Options" menu allows the user to specify a particular folder in which the program will look for input files and will write the output files. This is particularly useful when doing several analyses using different input factors or conducting sensitivity analysis. Choosing the "Set Data Directory" option causes the following dialog box to be displayed: 


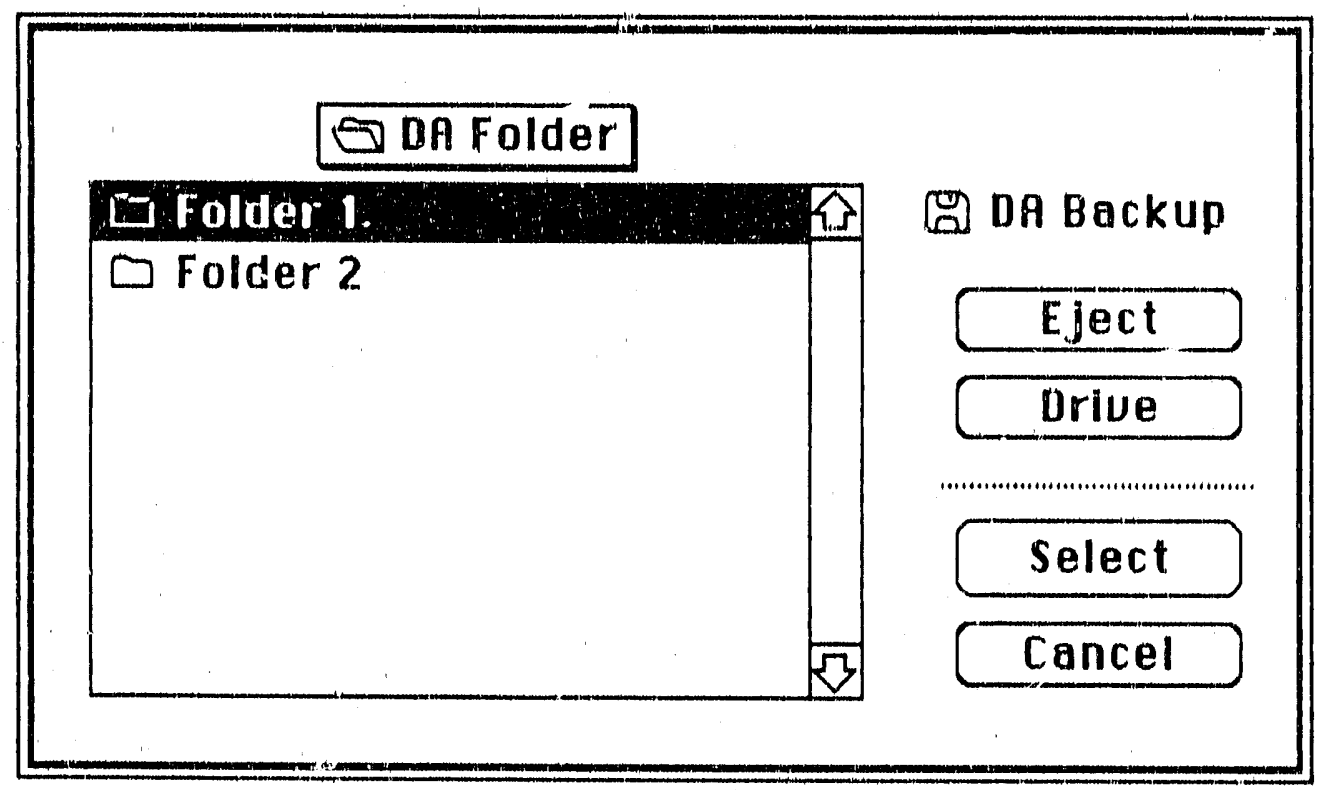

If the desired data folder is not in the current folder, the user can change the default drive, choose different folders on the current drive, or open folders within the current folder, until the desired data folder is displayed. Selecting the desired data folder and then clicking on the "Select" button causes the selected data folder to be made the data directory. Note that double-clicking on the desired folder will not set the data directory. If the "Set Data Directory" function is not used, the program will look for data files in the folder containing the application.

Each of the iterns on the menu under "Analysis" corresponds to one of the components of the model. Selecting one of these items will cause the corresponding component to be run. The program will display a dialog box prompting the user to specify needed parameter values. In some cases, default values are displayed. This dialog box also displays default names for any input files needed by the component. These names are: "SV Data," for the file containing threshold/violation data, "Evasion Data," for the Evasion Potential file, "F-factor," for the F-factor file, and "Utility File," for the Utility input file. The user can change the file names in this dialog box if files with other names are to be used. If the program is unable to find a specified file in the data directory, or if the specified file exists but is empty, the user will be prompted (via another dialog box) to specify a file that has the needed data. With the exception of the Cavity Decoupling component, each dialog box contains a square labelled "Evasion." Clicking on this box causes the program to prompt the user for an evasion file to be used as input. The default file name is "Evalsion Data." The dialog box also allows the user to specify a legend label for each case analyzed. This is used to label the resulting curve in the output graph, making multiple curves plotted on the same graph easier to distinguish. In addition to being used to label curves, these labels are also used as column headers in the tabular output files. In the Cavity Decoupling and Expected Utility components, the dialog box also asks for the name of an output file for each case run, and, in the case of evasion, a comment to go in that file. Default names for these files are "Evasion Data" and "Utility File." The 'dialog boxes that prompt the user for input data values and input file names are displayed below for each model component. The component name is in the lower right hand corner of each dialog box. 

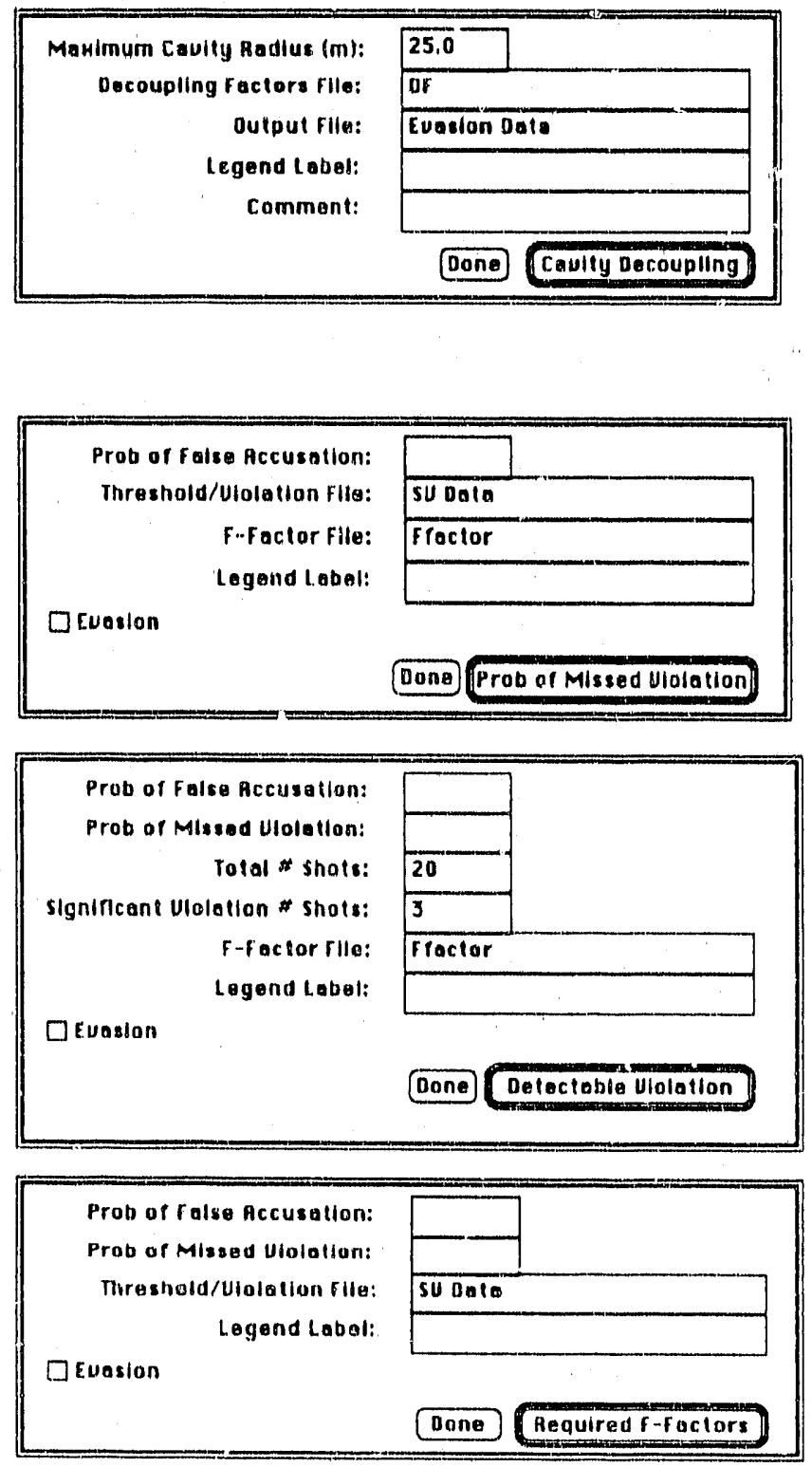
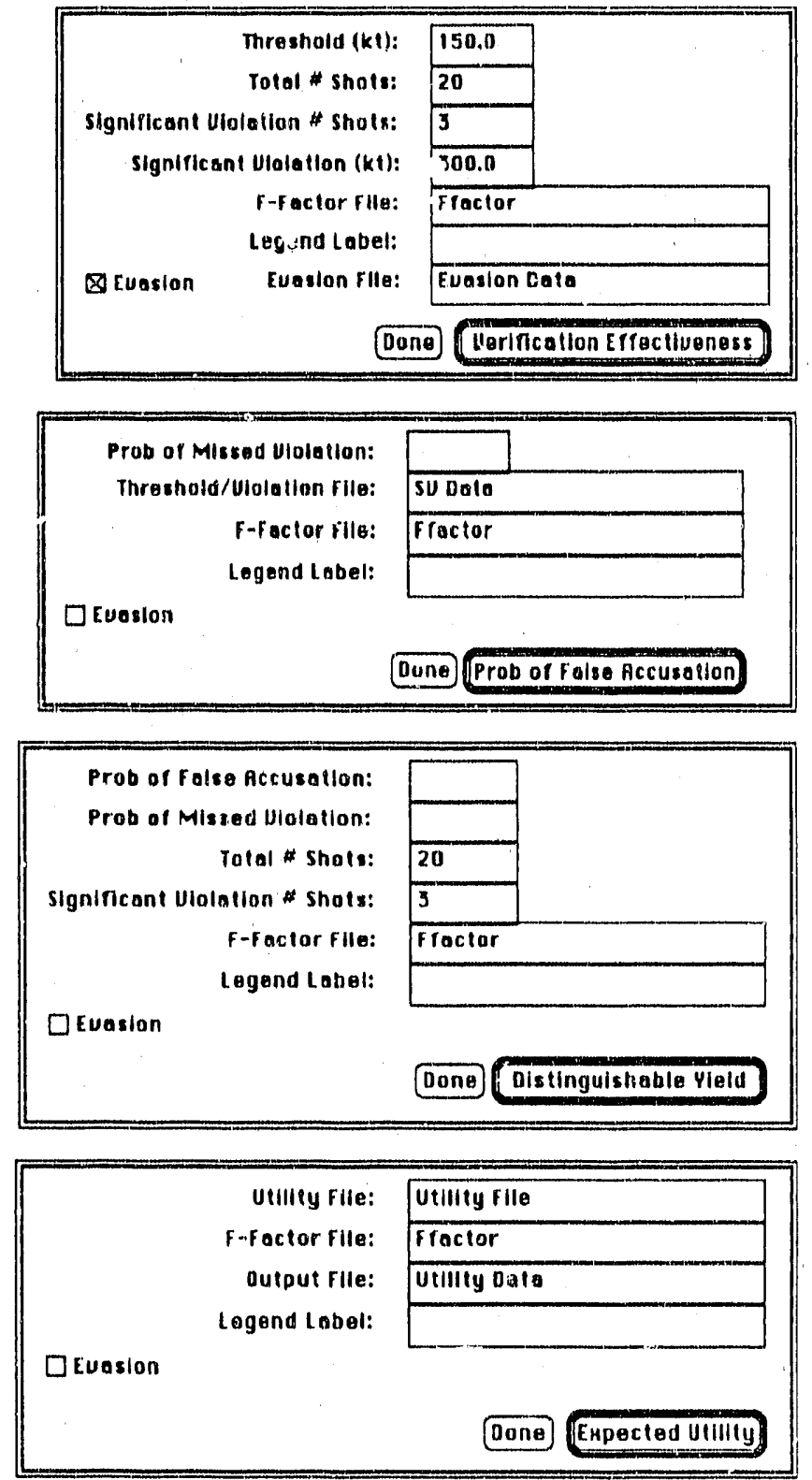

When all data inputs have been supplied, the user may start the calculations by clicking on the button that is labelled with the name of the model component. (These buttons, and others with double outlines, are default buttons. Pressing the return key causes the prograrn to act as though the default button had been clicked). The program will then perform the calculations and display the resulting graph, labelling the new curve with the label that was specified in the dialog box. A button labelled "Continue" will also be displayed. Clicking on this bution brings up the previous dialog box. The user can then choose whether to run additional cases (up to a maximum of five cases on the same graph) or to exit the component.

When all desired cases have been run, the user clicks on the "Done" button. Doing this brings up the following dialog box: 


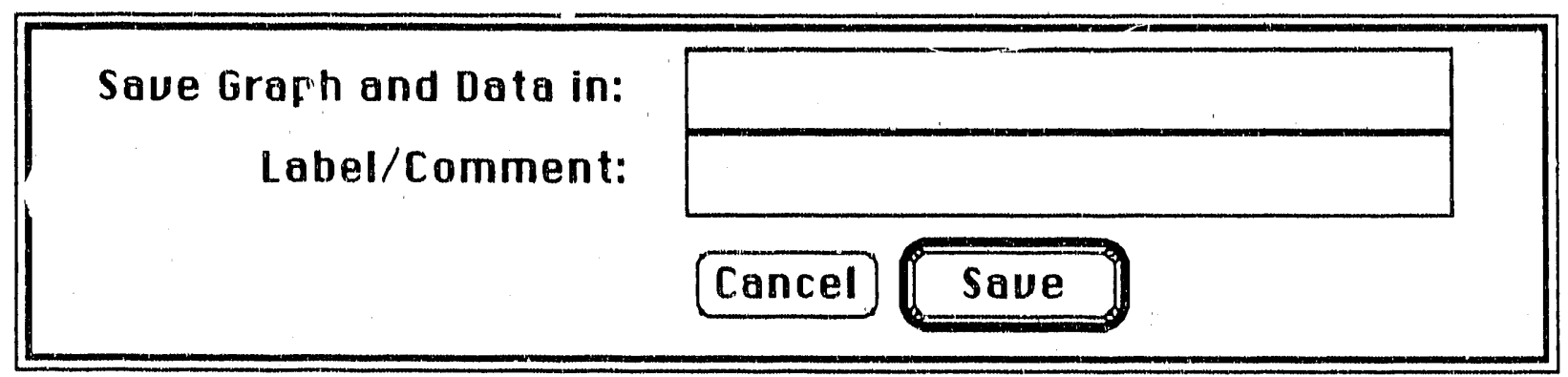

The user can either specify the name of a file in which to save the graph and data and then click on "Save," or click on the "Cancèl" button. The model uses the filename entered in this dialog box, together with the word "Graph" or the word "Data" as the filename of the graphical or tabular output file, respectively. Any text placed in the Label/Comment box is placed in the first line of the tabular output file and is used as a title on the graph that is saved. This dialog box is used by every component of the model except the Cavity Decoupling component. Since the tabular data from each run of the Cavity Decoupling zomponent is placed in a separate file, while several cases may be ploited on the same graph, the Cavity Decoupling component displays the following dialog box:

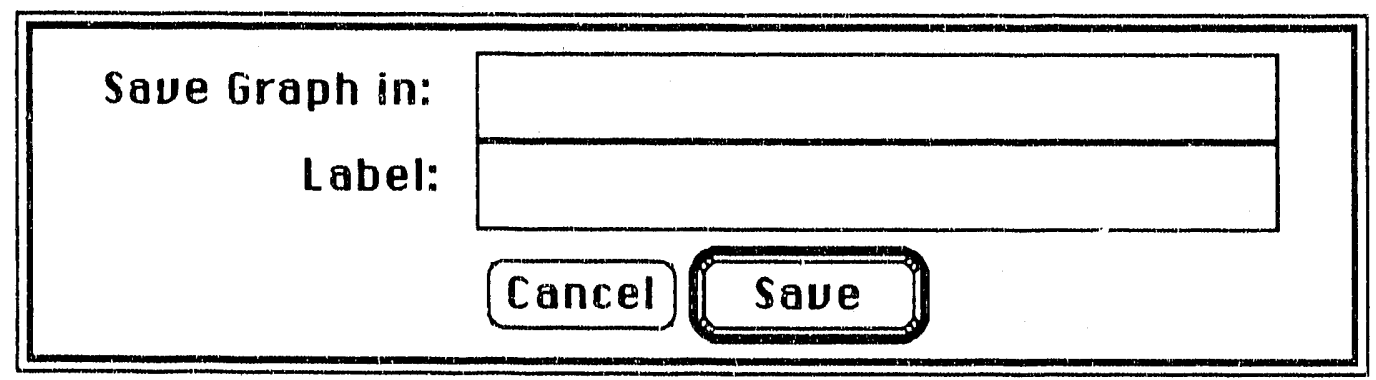

After clicking on either the "Save" or "Cancel" button, control is returned to the main menus and the user may set a new data directory ("Options" menu), run another component of the model ("Analysis" menu), or quit from the application ("File" menu). 


\section{Appendix B: Formats of Input and Output Files}

\section{Input Files}

The input files for the rnodel include the Decoupling Factor File, the Evasion Potential File, the F-factor File, the Threshold/Violation File, and the Utility File. These files may be prepared using any word-processing or spreadsheet software that allows a file to be saved as "text." Each of the input files has a similar format. The first line of each file is reserved for a comment. The second line contains column headings, and subsequent lines contain rows of data, with the fields in each row being separated by tabs. The files have the following formats:

\section{Decoupling Factor File}

Each row of data in this file consists of a scaled radius (in meters) in the first field and the corresponding decoupling factor in the second field.

\section{Evasion Potential File}

Each row of data in this file consists of an actual yield in the first field and the corresponding apparent yield in the second field. (For evasion by cavity decoupling, this file can be created using the cavity decoupling component.)

\section{F-factor File}

Each row of data in this file consists of a yield level in the first field ana the corresponding F-factor in the second field.

\section{Threshold/Violation File}

Each row of data in this file consists of a threshold in the first field, a violation yield in the second field, the number of tests at the violation yield that constitute a significant violation in the third field, and the number of tests at the threshold for the compliance scenario in the fourth field.

\section{Utility File}

For each row of data in this file, the first four fields are the same as in a threshold/violation file. The fifth field contains the prior probability that the Soviets will comply with the given threshold. Fields six through nine contain utility values for accepted compliance, false accusation, missed violation, and challenged violations, respectively. These values will ordinarily be on a scale from 0 to 100 . 


\section{Output Files}

The output files iriclude two types of files: graphical, which can be read by such software as MacPaint or SuperPaint, and tabular, which can be read by any software that accepts "text" files. Each component of the model produces a graph of up to five cases and allows the user to save it in a graphics file. The data used to generate the graphs may be saved in tabular files. With the exception of the Cavity Decoupling and Expected Utility components, each component of the model produces a single tabular file containing data from all of the cases analyzed. The Cavity Decoupling and Expected Utility components produce a tabular file for each case. The default names of these files are "Evasion Data" and "Utility Data," respectively. The former is also the default name when an evasion file is needed as input. The user must specify names for all other output files. The Expected Utility component can also produce a file which contains all of the data used to plot its graph.

For each tabular output file, the first line is reserved for a comment. The second line contains column headings, which are the labels given to each case by the user, and subsequent lines contain data. Each row of data consists of a field containing a value of the independent variable (the variable on the horizontal axis of the graph), followed by a field containing the computed value of the dependent variable. If the file contains output from more than one case, there will be several fields containing values of the dependent variable, one for each case. If the values of the independent variable are read from an input file, and more than one input file is to be used for the same graph, the values of the independent variable need not be the same in both input files. If this is the case, the first column of the output file will contain all the values of the independent variable that were used in any of the cases. Wherever a case did not use a particular value of the independent variable, the corresponding field in the output file will be blank. The fields in each row of data are separated by tabs. If spreadsheet software is used to view the file, each field will appear in a separate column. The tabular files produced by the various components have the following formats:

\section{Cavity Decoupling}

The format of this file has been described under the "Evasion Potential" heading in the input file section. Each row of data consists of an actual yield in the first field and the corresponding apparent yield in the second field. An individual file is produced for each case analyzed.

\section{Verification Effectiveness}

Each row of data consists of a probability of false accusation in the first field and the probabilities of missed violation for each case in the remaining fields. The false accusation probabilities used are $0.01,0.05,0.1,0.2, \ldots, 0.9,0.95$, and 0.99 .

\section{Probability of Missed Violation}

Each row of data consists of a threshold in the first field and the probability of missed violation for each case in the remaining fields. The threshold options used are read from the threshold/violation file. 


\section{Probability of False Accusation}

Each row of data in this file consists of a threshold in the first ficid and the probability of false accusation for each case in the remaining fields. The threshold options used are read from the threshold/violation file.

\section{Detectable Violation}

Each row of data in this file consists of a threshold in the first field and the detectable violation yield for each case in the remaining fields. The thresholds used are $5,10,15, \ldots$, and $150 \mathrm{kt}$.

\section{Distinguishable Yield}

Each row of data consists of a violation yield in the first field and the distinguishable threshold for each case in the remaining fields. The violation yields used are $10,20,30, \ldots$, an. $300 \mathrm{kt}$.

\section{Required Monitoring Capability}

Each row of data consists of a threshold in the first field and the required F-factor for each case in the remaining fields. The thresholds options used are read from the threshold/violation file.

\section{Expected Utility}

Two types of files are produced by the Expected Utility component. A file of the first type is produced for each case run. Each row of data in these files consists of five fields. The first field contains a threshold, the second field contains the expected overall utility of that threshold, the third field contains the optimal decision line, which results in the computed expected utility, and the fourth and fifth fields contain the probabilities of missed violation and false accusation that result from the optimal decision line.

The second type of file contains the data used to generate the graph. The rows of data in this file consist of a threshold in the first field and the expected utility of that threshold for each case in the remaining fields. 

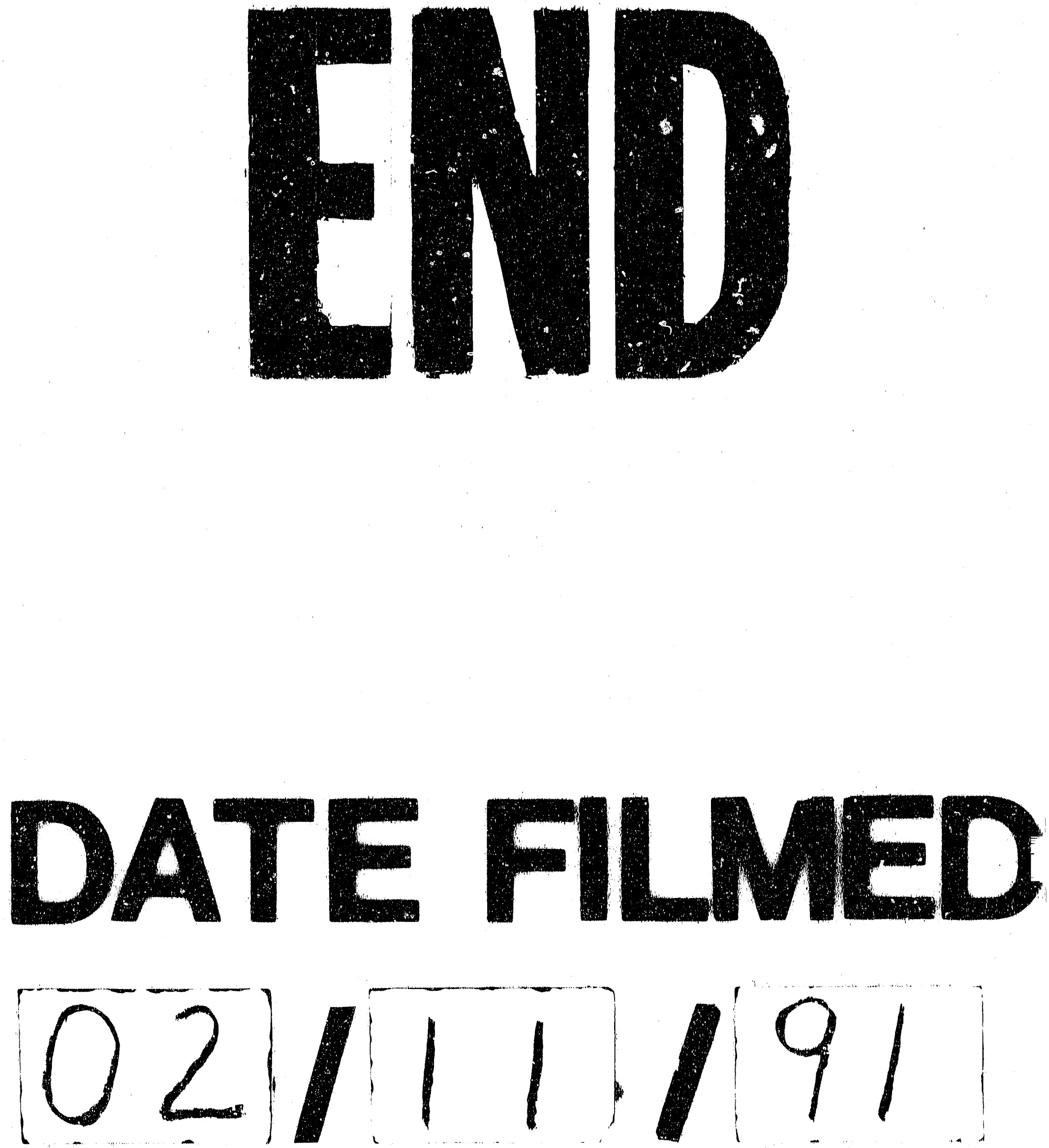
